\title{
Maximal Subsemigroups containing a particular semigroup
}

\author{
Jörg Koppitz ${ }^{\dagger}$, Tiwadee Musunthia $\sharp$
}

\author{
Potsdam University, ${ }^{\dagger}$ \\ Institute of Mathematics \\ Am Neuen Palais, D-14415 \\ Potsdam, Germany
}

e-mail: koppitz@rz.uni-potsdam.de

\author{
Department of Mathematics ${ }^{\sharp}$ \\ Silpakorn University \\ Nakorn Pathom, Thailand 73000 \\ Centre of Excellence in Mathematics \\ 272 Rama VI Road, Bangkok \\ 10400, Thailand \\ e-mail: tiwadee_m@hotmail.com
}

\begin{abstract}
We study maximal subsemigroups of the monoid $T(X)$ of all full transformations on the set $X=N$ of natural numbers containing a given subsemigroup $W$ of $T(X)$ where each element of a given set $U$ is a generator of $T(X)$ modulo $W$. This note continue the study of maximal subsemigroups on the monoid of all full transformations on a infinite set.
\end{abstract}

In this note, we want to continue the study of maximal subsemigroups of the semigroup $T(X)$ of all full transformations on an infinite set, in particular, for the case $X$ is countable. The maximal subsemigroups of $T(X)$ containing the symmetric group $\operatorname{Sym}(X)$ of all bijective mappings on an infinite set are already known. They were determined by L. Heindorf ( $X$ is countable) and by M. Pinsker (any infinite set $X$ ) characterizing maximal clones $([3,[6])$.

The setwise stabilizer of any finite set $Y \subseteq X$ under $\operatorname{Sym}(X)$ is a subgroup of $\operatorname{Sym}(X)$. In [1], the authors determine the maximal subsemigroups of $T(X)$ containing the setwise stabilizer of any finite set $Y \subseteq X$ under $\operatorname{Sym}(X)$. For a finite partition of $X$, one can also consider the (almost) stabilizer. They form subsemigroups of $\operatorname{Sym}(X)$ and in [1, the maximal subsemigroups of $T(X)$ containing such a subgroup are determined. Also in [1], the maximal subsemigroups containing the stabilizer of any uniform ultrafilter on $X$, which forms also a group, are determined.

In the present note, we consider a countable infinite set $X$ and characterize for a given subsemigroup $W$ of $T(X)$ and a given set $U \subseteq T(X)$, where any element of $U$ is a generator modulo $W$ (see [4]), the maximal subsemigroups of $T(X)$ containing $W$. As a consequence of this result, we obtain all maximal subsemigroups of $T(X)$ containing $T(X) \backslash S$, where $S$ is a given maximal subsemigroup of $T(X)$ containing $\operatorname{Sym}(X)$.

Notation 1. Let $M \subseteq P(T(X))$ and let $J(M)$ be the set of all $A \subseteq T(X)$ with

$A \subseteq \bigcup M:=\{a \mid \exists m \in M \wedge a \in m\}$,

$A \cap m \neq \emptyset$ for all $m \in M$, and

$\forall \alpha \in A \exists m \in M$ with $A \cap m=\{\alpha\}$.

Definition 2. Let $U \subseteq T(X)$ and $W \leq T(X)$. Then we put

$\operatorname{Gen}(U):=\{A \subseteq T(X) \mid A$ is finite and $\langle A\rangle \cap U \neq \emptyset\}$ and

$\mathcal{H}(U, W):=\{A \subseteq T(X) \backslash W \mid A \in J(G e n(U))\}$. 
Theorem 3. Let $W \leq S \leq T(X)$ and $U \subseteq T(X)$ with $U \cap W=\emptyset$ such that $\langle W, \alpha\rangle=$ $T(X)$ for all $\alpha \in U$. Then the following statements are equivalent:

(i) $S$ is maximal.

(ii) There is a $H \in \mathcal{H}(U, W)$ with $S=T(X) \backslash H$.

Proof. $(i) \Rightarrow($ ii $)$ : Assume that $S \cap H \neq \emptyset$ for all $H \in \mathcal{H}(U, W)$. Then there is $A \in$ $\operatorname{Gen}(U)$ with $A \subseteq S$. Otherwise $A \nsubseteq S$, i.e. $A \backslash S \neq \emptyset$, for all $A \in G e n(U)$. We consider the set $\operatorname{Gen}(U)=\left\{A_{i} \mid i \in \mathbb{N}\right\}$. We put $\bar{A}_{i}=A_{i} \backslash S$ for all $i \in \mathbb{N}$,

$H_{0}=\emptyset$ and $\bar{H}_{0}=\emptyset$;

$H_{i+1}=H_{i}$ if $\bar{A}_{i+1} \cap H_{i} \neq \emptyset$ or $\exists j>i+1$ with $\bar{A}_{j} \backslash \bar{H}_{i} \subseteq \bar{A}_{i+1}$ then we define

$\bar{H}_{i+1}=\bar{H}_{i}$;

$H_{i+1}=H_{i} \cup\left\{a_{i+1}\right\}$ if $\bar{A}_{i+1} \cap H_{i}=\emptyset$ and $\forall j>i+1, \bar{A}_{j} \backslash \bar{H}_{i} \nsubseteq \bar{A}_{i+1}$ then we define

$\bar{H}_{i+1}=\left(\bar{H}_{i} \cup \bar{A}_{i+1}\right) \backslash\left\{a_{i+1}\right\}$ for $a_{i+1} \in \bar{A}_{i+1} \backslash \bar{H}_{i}$.

We want to show that $\bar{A}_{i+1} \backslash \bar{H}_{i} \neq \emptyset$ for all $i \in \mathbb{N}$. Let us consider $i \in \mathbb{N}$. We know that $\bar{A}_{i}=A_{i} \backslash S \neq \emptyset$ by assumption. If $H_{i}=\emptyset$ then $\bar{A}_{i+1} \backslash \bar{H}_{i} \neq \emptyset$. Assume that $H_{i} \neq \emptyset$. Then there exists $k \in \mathbb{N}$ with $k<i$ such that $H_{k} \cup\left\{a_{k+1}\right\}=H_{k+1}=H_{i}$. If $\bar{A}_{i+1} \backslash \bar{H}_{k} \subseteq \bar{A}_{k+1}$ then $\bar{H}_{k+1}=\bar{H}_{k}=\bar{H}_{i}$. This implies that $\bar{A}_{i+1} \backslash \bar{H}_{i} \neq \emptyset$. Then $\bar{A}_{j} \backslash \bar{H}_{k} \nsubseteq \bar{A}_{k+1}$ for all $j>k+1$. Thus $\bar{A}_{i+1} \backslash \bar{H}_{k} \nsubseteq \bar{A}_{k+1}$ and there exists $x \in \bar{A}_{i+1}$ but $x \notin \bar{H}_{k}$ and $x \notin \bar{A}_{k+1}$, i.e. $x \in \bar{A}_{i+1}$ but $x \notin \bar{H}_{k} \cup \bar{A}_{k+1}$. Moreover, we have $x \in \bar{A}_{i+1}$ but $x \notin\left(\bar{H}_{k} \cup \bar{A}_{k+1}\right) \backslash\left\{a_{k+1}\right\}=\bar{H}_{i}$. This shows that $x \in \bar{A}_{i+1} \backslash \bar{H}_{i}$. This completes the proof that $\bar{A}_{i+1} \backslash \bar{H}_{i} \neq \emptyset$. Moreover, we put $H:=\bigcup_{i \in \mathbb{N}} H_{i}$.

We want to show that $H:=\bigcup_{i \in \mathbb{N}} H_{i} \in J(G e n(U))$. First, we will show that $H \subseteq$ $\bigcup G e n(U):=\left\{a_{i} \mid \exists A_{i} \in G e n(U) \wedge a_{i} \in A_{i}\right\}$. By definition of $H$, we know that $H_{0}=\emptyset$, $H_{i+1}=H_{i}$ or $H_{i+1}=H_{i} \cup\left\{a_{i+1}\right\}$ for $i \in \mathbb{N}$. This shows that $H \subseteq \bigcup G e n(U)$.

Let $i \in \mathbb{N}$. Then we have to show that $H \cap A_{i} \neq \emptyset$. If $a_{i} \in H$ then all is clear. Otherwise, assume that $a_{i} \notin H$. Then, $\bar{A}_{i} \cap H_{i-1} \neq \emptyset$ and thus $\bar{A}_{i} \cap H_{i} \neq \emptyset$ or $\exists k>i$ with $\bar{A}_{k} \backslash \bar{H}_{i-1} \subseteq \bar{A}_{i}$. We have to consider $\bar{A}_{k}$ with $k>i$. Then $a_{k} \in H$ or we have the same cases as for $A_{i}$. Since the elements of $\operatorname{Gen}(U)$ are finite, this procedure finish. So we obtain an $s \geqq i$ such that $a_{s} \in H$ and it is routine to see that also $a_{s} \in \bar{A}_{i}$. Thus $H \cap A_{i} \neq \emptyset$.

Let $a \in H$. Then there exists an $i \in \mathbb{N}$ such that $a=a_{i} \in A_{i}$. We have to show that for all $k \in \mathbb{N} \backslash\{i\}$, if $a_{k} \in H$ (i.e. $a_{k} \in A_{k}$ ) then $a_{k}$ does not belong to $A_{i}$. We have two cases:

Case 1: $k>i$. Then $a_{k}$ does not belong to $H_{i} \subseteq H_{k-1}$ and moreover, it does not belong to $\bar{H}_{i} \subseteq \bar{H}_{k-1}$. But $\bar{A}_{i} \subseteq H_{i} \cup \bar{H}_{i}$. So $a_{k}$ does not belong to $A_{i}$.

Case 2: $k<i$. Then $a_{k} \in H_{i-1}$ where $H_{i-1} \cap A_{i}=\emptyset$, whence $a_{k} \notin A_{i}$.

This shows that $H \in J(G e n(U))$. But $H \cap S=\emptyset$ is a contradiction. Then there is $A \in G e n(U)$ with $A \subseteq S$, i.e. $\langle A\rangle \cap U \neq \emptyset$. Let $\alpha \in\langle A\rangle \cap U \subseteq S$. Then $T(X)=\langle W, \alpha\rangle \leq$ $S$, i.e. $S=T(X)$, a contradiction. Hence, there is $H \in \mathcal{H}(U, W)$ with $S \cap H=\emptyset$, i.e. $S \subseteq T(X) \backslash H$. We want to show that $T(X) \backslash H$ is a semigroup. Let $\alpha, \beta \in T(X) \backslash H$. Assume that $\alpha \beta \in H$. Then there is $A \in \operatorname{Gen}(U)$ with $A \cap H=\{\alpha \beta\}$. Since $U \cap\langle A\rangle \neq \emptyset$, $U \cap\langle A \backslash\{\alpha \beta\} \cup\{\alpha, \beta\}\rangle \neq \emptyset$, i.e. $(A \backslash\{\alpha \beta\}) \cup\{\alpha, \beta\} \in G e n(U)$. Since $A \cap H=\{\alpha \beta\}$, $(A \backslash\{\alpha \beta\}) \cup\{\alpha, \beta\} \in G e n(U)$, implies $\alpha \in H$ or $\beta \in H$, a contradiction. Hence $T(X) \backslash H$ 
is a semigroup. Since $S$ is a maximal subsemigroup of $T(X)$ and $S \subseteq T(X) \backslash H$ this implies $S=T(X) \backslash H$.

$(i i) \Rightarrow(i)$ : Let $H \in \mathcal{H}(U, W)$ with $S=T(X) \backslash H$. We have shown that $T(X) \backslash H$ is a semigroup. Now, we want to show that it is a maximal subsemigrop of $T(X)$. Let $\alpha \in H$. Then there is $A \in G e n(U)$ with $A \cap H=\{\alpha\}$ and $T(X)=\langle W, A\rangle \subseteq$ $\langle T(X) \backslash H, A\rangle=\langle T(X) \backslash H, \alpha\rangle$ since $\langle A\rangle \cap U \neq \emptyset$ and $\langle W, \beta\rangle=T(X)$ for all $\beta \in \bar{U}$. So, $\langle T(X) \backslash H, \alpha\rangle=T(X)$. This shows that $T(X) \backslash H$ is a maximal subsemigroup of $T(X)$.

If $\alpha \in T(X)$ and $A \subseteq X$ such that the restriction of $\alpha$ to $A$ is injective and have the same range as $\alpha$, then we will refer $A$ as transversal of $\alpha$ (ker $\alpha$ denotes the kernel of $\alpha$ ). We will also write $A \# \operatorname{ker} \alpha$ if $A$ is a transversal of $\alpha$.

Let $D(\alpha):=X \backslash i m \alpha(i m \alpha$ denotes the range of $\alpha)$. The rank $\alpha$, i.e. the cardinality of $i m \alpha$, is denoted by $\operatorname{rank}(\alpha):=|i m \alpha|$. Then $d(\alpha):=|D(\alpha)|$ is called defect of $\alpha$ and $c(\alpha):=\sum_{y \in i m \alpha}\left(\left|y \alpha^{-1}\right|-1\right)$ is called collapse of $\alpha$.

Moreover, we put $K(\alpha):=\left\{x \in i m \alpha|| x \alpha^{-1} \mid=\aleph_{0}\right\}$ and $k(\alpha):=|K(\alpha)|$ is called infinite contractive index. It is well known that $d(\alpha \beta) \leq d(\alpha)+d(\beta), k(\alpha \beta) \leq k(\alpha)+k(\beta)$ [2] and $c(\alpha \beta) \leq c(\alpha)+c(\beta)$ [?] for $\alpha, \beta \in T(X)$. For more background in the theory of transformation semigroups see [2] and [5].

Now we want to determine the maximal subsemigroups of $T(X)$ containing $T(X) \backslash S$, where $S$ is one of the five maximal subsemigroups of $T(X)$ containing $\operatorname{Sym}(X)$. Let us introduce the following five sets:

- $\operatorname{Inj}(X):=\left\{\alpha \in T(X) \mid \operatorname{rank}(\alpha)=\aleph_{0}, c(\alpha)=0\right.$ and $\left.d(\alpha) \neq 0\right\}$ (the set of injective but not surjective mappings on $X)$.

- $\operatorname{Sur}(X):=\left\{\alpha \in T(X) \mid \operatorname{rank}(\alpha)=\aleph_{0}, c(\alpha) \neq 0\right.$ and $\left.d(\alpha)=0\right\}$ (the set of surjective but not injective mappings on $X)$.

- $C_{p}(X):=\left\{\alpha \in T(X) \mid \operatorname{rank}(\alpha)=\aleph_{0}, k(\alpha)=\aleph_{0}\right\}$.

- $I F(X):=\left\{\alpha \in T(X) \mid \operatorname{rank}(\alpha)=\aleph_{0}, c(\alpha)=\aleph_{0}\right.$ and $\left.d(\alpha)<\aleph_{0}\right\}$.

- $F I(X):=\left\{\alpha \in T(X) \mid \operatorname{rank}(\alpha)=\aleph_{0}, d(\alpha)=\aleph_{0}\right.$ and $\left.c(\alpha)<\aleph_{0}\right\}$.

In [3], the following proposition was proved. Note that we independently proved this proposition whilst of the work of L. Heindorf. We thank Martin Goldstern for bringing these reference to our consideration at the AAA82 in Potsdam (June 2011). For the sake of completeness, we include the proof of this proposition.

Proposition 4. The following semigroups of $T(X)$ are maximal:

$$
T(X) \backslash H
$$

for $H \in\left\{\operatorname{Inj}(X), \operatorname{Sur}(X), C_{p}(X), I F(X), F I(X)\right\}$. 
Proof. 1) Let $\alpha, \beta \in T(X) \backslash \operatorname{Inj}(X)$. Assume that $\alpha \beta \in \operatorname{Inj}(X)$. Then $c(\alpha)=0$, i.e. $\alpha$ is injective. Since $\alpha \notin \operatorname{Inj}(X), \alpha \in \operatorname{Sym}(X)$. But $c(\alpha \beta)=0$ and $\alpha \in \operatorname{Sym}(X)$ implies $\beta$ is injective. Since $\beta \notin \operatorname{Inj}(X), \beta \in \operatorname{Sym}(X)$. So $\alpha \beta \in \operatorname{Sym}(X)$, i.e. $\alpha \beta$ is surjective, contradicts $\alpha \beta \in \operatorname{Inj}(X)$. This shows that $T(X) \backslash \operatorname{Inj}(X)$ is a semigroup.

Let $\alpha \in \operatorname{Inj}(X)$. Then we will show that $\langle T(X) \backslash \operatorname{Inj}(X), \alpha\rangle=T(X)$. For this let $\beta \in \operatorname{Inj}(X)$. Let $a \in i m \beta$. Let $\gamma \in T(X)$ with $x \gamma=a$ for $x \in D(\alpha)$, and $x \gamma=x \alpha^{-1} \beta$ for $x \in i m \alpha$. Then $x \alpha \gamma=x \alpha \alpha^{-1} \beta=x \beta$ for all $x \in X$. This shows $\alpha \gamma=\beta$, where $\gamma \notin \operatorname{Inj}(X)$ since $D(\alpha) \neq \emptyset$. This shows that $T(X) \backslash \operatorname{Inj}(X)$ is maximal.

2) Let $\alpha, \beta \in T(X) \backslash \operatorname{Sur}(X)$. Assume that $\alpha \beta \in \operatorname{Sur}(X)$. Then $d(\beta)=0$, i.e. $\beta$ is surjective. Since $\beta \notin \operatorname{Sur}(X), \beta \in \operatorname{Sym}(X)$. But $d(\alpha \beta)=0$ and $\beta \in \operatorname{Sym}(X)$ implies $\alpha$ is surjective. Since $\alpha \notin \operatorname{Sur}(X), \alpha \in \operatorname{Sym}(X)$. So $\alpha \beta \in \operatorname{Sym}(X)$, i.e. $\alpha \beta$ is injective, contradicts $\alpha \beta \in \operatorname{Sur}(X)$. This shows that $T(X) \backslash \operatorname{Sur}(X)$ is a semigroup.

Let $\alpha \in \operatorname{Sur}(X)$. Then we will show that $\langle T(X) \backslash S u r(X), \alpha\rangle=T(X)$. For this let $\beta \in$ $\operatorname{Sur}(X)$. For all $\bar{x} \in X / \operatorname{ker} \alpha$ we fix a $\bar{x}^{*} \in \bar{x}$. Then we consider the following $\delta \in T(X)$ with $i \delta=\left(i \beta \alpha^{-1}\right)^{*}$ for all $i \in X$. Hence $i \delta \alpha=i \beta$ for all $i \in X$. This shows $\delta \alpha=\beta$. Since $i m \delta=\left\{\bar{x}^{*} \mid \bar{x} \in X / \operatorname{ker} \alpha\right\} \neq X$ (because $\alpha$ is not injective), $\delta \in T(X) \backslash \operatorname{Sur}(X)$. This shows $\beta=\delta \alpha \in\langle T(X) \backslash \operatorname{Sur}(X), \alpha\rangle$. Consequently, $\langle T(X) \backslash \operatorname{Sur}(X), \alpha\rangle=T(X)$. This shows that $T(X) \backslash \operatorname{Sur}(X)$ is maximal.

3) Let $\alpha, \beta \in T(X) \backslash C_{p}(X)$. Further, let $x \in i m \alpha \beta$. Then $x(\alpha \beta)^{-1}=\left(x \beta^{-1}\right) \alpha^{-1}$ and $\left|\left(x \beta^{-1}\right) \alpha^{-1}\right|=\aleph_{0}$ if $\left|x \beta^{-1}\right|=\aleph_{0}$ or there is a $y \in x \beta^{-1} \cap i m \alpha$ with $\left|y \alpha^{-1}\right|=\aleph_{0}$. This shows $k(\alpha \beta) \leq k(\alpha)+k(\beta)<\aleph_{0}+\aleph_{0}=\aleph_{0}$. This shows that $\alpha \beta \in T(X) \backslash C_{p}(X)$.

Let $\alpha \in C_{p}(X)$. Then, we will show that $\left\langle T(X) \backslash C_{p}(X), \alpha\right\rangle=T(X)$. For this let $\beta \in C_{p}(X)$. Then, there is a bijection

$$
f: X / \operatorname{ker} \beta \rightarrow\left\{x \alpha^{-1} \mid x \in K(\alpha)\right\}
$$

For each $\bar{x} \in X / \operatorname{ker} \beta$, there is an injective mapping

$$
f_{\bar{x}}: \bar{x} \rightarrow f(\bar{x}) .
$$

We take the $\gamma \in \operatorname{Inj}(X)$ with $i \gamma=f_{\bar{x}}(i)$ where $i \in \bar{x}$ for $\bar{x} \in X / \operatorname{ker} \beta$. For $i, j \in X$, $i \beta=j \beta$ if and only if there is an $\bar{x} \in X / \operatorname{ker} \beta$ with $i, j \in \bar{x}$, i.e. $f_{\bar{x}}(i) \alpha=f_{\bar{x}}(j) \alpha$. But $f_{\bar{x}}(i) \alpha=f_{\bar{x}}(j) \alpha$ is equivalent to $i \gamma \alpha=j \gamma \alpha$, consequently, we have $i \gamma \alpha=j \gamma \alpha$ if and only if $i \beta=j \beta$. Further, let $\delta \in T(X)$ with $i \gamma \alpha \delta=i \beta$ for $i \in X$ and $i \delta=x_{0}\left(x_{0}\right.$ is any fixed element in $X)$ for $i \in X \backslash i m \gamma \alpha$. Since $i \gamma \alpha=j \gamma \alpha$ if and only if $i \beta=j \beta, \delta$ is well defined. Moreover, $\left|\left\{\bar{x}|\bar{x} \in X / \operatorname{ker} \delta,| \bar{x} \mid=\aleph_{0}\right\}\right|<2$, i.e. $\delta \in T(X) \backslash C_{p}(X)$. This shows $\beta=\gamma \alpha \delta \in\left\langle T(X) \backslash C_{p}(X), \alpha\right\rangle$. Consequently, $\left\langle T(X) \backslash C_{p}(X), \alpha\right\rangle=T(X)$. This shows that $T(X) \backslash C_{p}(X)$ is maximal.

4) Let $\alpha, \beta \in T(X) \backslash I F(X)$.

If $c(\alpha)<\aleph_{0}$ and $c(\beta)<\aleph_{0}$ then $c(\alpha \beta) \leq c(\alpha)+c(\beta)<\aleph_{0}$, i.e. $\alpha \beta \notin I F(X)$.

If $d(\alpha)=\aleph_{0}$ and $c(\beta)<\aleph_{0}$ then $|\{\bar{x} \in X / \operatorname{ker} \beta \mid \bar{x} \cap i m \alpha=\emptyset\}|=\aleph_{0}$. This implies $d(\alpha \beta)=\aleph_{0}$, i.e. $\alpha \beta \notin I F(X)$.

If $d(\beta)=\aleph_{0}$ then $d(\alpha \beta) \geq d(\beta)=\aleph_{0}$, i.e. $\alpha \beta \notin I F(X)$.

Altogether, this shows that $\alpha \beta \in T(X) \backslash I F(X)$.

Let $\alpha \in I F(X)$. Then we will show that $\langle T(X) \backslash I F(X), \alpha\rangle=T(X)$. For this let $\beta \in I F(X)$. Let $\gamma \in T(X)$ with $\operatorname{ker} \gamma=\operatorname{ker} \beta$ and $i m \gamma \# \operatorname{ker} \alpha$. For each $\bar{x} \in X / \operatorname{ker} \beta$, 
we fix any $\bar{x}^{*}$. Since $c(\alpha)=\aleph_{0}, d(\gamma)=\aleph_{0}$, i.e. $\gamma \notin I F(X)$. Further, let $\delta \in T(X)$ with $i m \alpha \# \operatorname{ker} \delta$ and $i \delta=\left(i(\gamma \alpha)^{-1}\right)^{*} \beta$ for $i \in i m \alpha$. Since $i m \gamma \#$ ker $\alpha$, we have $i m \alpha=i m \gamma \alpha$ and $\delta$ is well defined. Because of $i m \gamma \# \operatorname{ker} \alpha, \operatorname{ker} \gamma \alpha=\operatorname{ker} \gamma=\operatorname{ker} \beta$, where $i \gamma \alpha \delta=$ $\left(i \gamma \alpha(\gamma \alpha)^{-1}\right)^{*} \beta=i \beta$ for $i \in X$. Note that from $i m \alpha \# \operatorname{ker} \delta$ and $d(\alpha)<\aleph_{0}$, it follows $c(\delta)<\aleph_{0}$, i.e. $\delta \notin I F(X)$. This shows $\beta=\gamma \alpha \delta \in\langle T(X) \backslash I F(X), \alpha\rangle$. Consequently, $\langle T(X) \backslash I F(X), \alpha\rangle=T(X)$. This shows that $T(X) \backslash I F(X)$ is maximal.

5) Let $\alpha, \beta \in T(X) \backslash F I(X)$.

If $c(\alpha)=\aleph_{0}$ then $c(\alpha \beta) \geq c(\alpha)=\aleph_{0}$, i.e. $\alpha \beta \notin F I(X)$.

If $d(\alpha)<\aleph_{0}$ and $c(\beta)=\aleph_{0}$ then $\mid\{i \in i m \alpha \mid \exists j \in i m \alpha \backslash\{i\}$ such that $i \beta=j \beta\} \mid=\aleph_{0}$. This implies $c(\alpha \beta)=\aleph_{0}$, i.e. $\alpha \beta \notin F I(X)$.

If $d(\alpha)<\aleph_{0}$ and $d(\beta)<\aleph_{0}$ then $d(\alpha \beta) \leq d(\alpha)+d(\beta)<\aleph_{0}$, i.e. $\alpha \beta \notin F I(X)$.

Altogether, this shows that $\alpha \beta \in T(X) \backslash F I(X)$.

Let $\alpha \in F I(X)$. Then, we will show that $\langle T(X) \backslash F I(X), \alpha\rangle=T(X)$. For this, let $\beta \in F I(X)$. Let $\gamma \in T(X)$ with $\operatorname{ker} \gamma=\operatorname{ker} \beta$ and $i m \gamma \# \operatorname{ker} \alpha$. For each $\bar{x} \in X / \operatorname{ker} \beta$, we fix any $\bar{x}^{*}$. Since $c(\alpha)<\aleph_{0}, d(\gamma)<\aleph_{0}$, i.e. $\gamma \notin F I(X)$. Further, let $\delta \in T(X)$ with $i m \alpha \# \operatorname{ker} \delta$ and $i \delta=\left(i(\gamma \alpha)^{-1}\right)^{*} \beta$ for $i \in i m \alpha$. Since $i m \gamma \# \operatorname{ker} \alpha$, im $\alpha=i m \gamma \alpha$ and $\delta$ is well defined, where $i \gamma \alpha \delta=\left(i \gamma \alpha(\gamma \alpha)^{-1}\right)^{*} \beta=i \beta$ for $i \in X$. Note that from $i m \alpha \# \operatorname{ker} \delta$ and $c(\alpha)=\aleph_{0}$, it follows $d(\delta)=\aleph_{0}$, i.e. $\delta \notin F I(X)$. This shows $\beta=$ $\gamma \alpha \delta \in\langle T(X) \backslash F I(X), \alpha\rangle$. Consequently, $\langle T(X) \backslash F I(X), \alpha\rangle=T(X)$. This shows that $T(X) \backslash F I(X)$ is maximal.

First, we characterize the maximal subsemigroups of $T(X)$ containing $\operatorname{Inj}(X)$ and $\operatorname{Sur}(X)$, respectively. Note that we do not need Theorem 3 here. It is well known that the set $F(X)$ of all transformation of a finite rank forms an ideal of $T(X)$ and $\operatorname{Inf}(X):=T(X) \backslash F(X)$ generates $T(X)$. The next lemma shows that any maximal subsemigroup $S$ of $T(X)$ has the form $S=F(X) \cup T$ for some $T \subset \operatorname{Inf}(X)$.

Lemma 5. Let $S$ be a maximal subsemigroup of $T(X)$. Then $F(X) \subset S$.

Proof. We have $\operatorname{Inf}(X) \nsubseteq S$ (since $S \neq T(X)$ ). Since $F(X)$ forms an ideal of $T(X)$ and thus $\operatorname{Inf}(X) \nsubseteq S$ implies $S \subseteq S \cup F(X) \neq T(X)$. Because of the maximality of $S$, we have $S=S \cup F(X)$, i.e. $F(X) \subset S$.

Lemma 6. Let $\operatorname{Sur}(X) \subset S \leq T(X)$ with $\operatorname{Inj}(X) \cap S \neq \emptyset$ and $F I(X) \cap S \neq \emptyset$. Then

$$
S=T(X) .
$$

Proof. We have $F(X) \subset S$ by Lemma 5. Hence, we have to consider only the elements of $\operatorname{Inf}(X)$. Let $\alpha \in \operatorname{Sym}(X)$. Then there is a $\beta \in \operatorname{Inj}(X) \cap S$ and we take the $\gamma \in \operatorname{Sur}(X)$ with $i \gamma=i$ for $i \in D(\beta)$ and $i \beta \gamma=i \alpha$ for $i \in X$. Since $i m \beta=X \backslash D(\beta)$, this shows that $\beta \gamma=\alpha$, and consequently, $\operatorname{Sym}(X) \subset S$. Let us put

$$
\begin{aligned}
& A:=\left\{\alpha \in \operatorname{Inf}(X) \mid d(\alpha)<\aleph_{0}\right\} \\
& B:=\left\{\alpha \in \operatorname{Inf}(X) \mid d(\alpha)=\aleph_{0}\right\}
\end{aligned}
$$


Clearly, $\operatorname{In} f(X)=A \cup B$. Let $\alpha \in A$. If $d(\beta)<\aleph_{0}$ then for each natural number $k \geq 1$, there is a natural number $r \geq 1$ such that $d\left(\beta^{r}\right) \geq k$. Since $\beta^{r} \in \operatorname{Inj}(X) \cap S$, we can assume that $d(\beta) \geq d(\alpha)$. Since $d(\beta) \geq d(\alpha)$, there is a $\gamma_{1} \in \operatorname{Sur}(X)$ such that $\gamma_{1}$ restricted to $i m \beta$ is bijective with $i m \alpha$ as range and $D(\beta) \gamma_{1}=D(\alpha)$. We take the $\gamma_{2} \in \operatorname{In} f(X)$ with $i \gamma_{2}$ is the unique element in $i \alpha \gamma_{1}^{-1} \beta^{-1}$ for $i \in X$. Since $\beta$ is injective, we have $\gamma_{2} \in \operatorname{Sur}(X) \cup \operatorname{Sym}(X)$. Then we have $i \gamma_{2} \beta \gamma_{1}=i \alpha \gamma_{1}^{-1} \beta^{-1} \beta \gamma_{1}=i \alpha$ for $i \in X$. This shows $\alpha=\gamma_{2} \beta \gamma_{1} \in S$, and consequently, $A \subset S$.

Let $\alpha \in B$. Moreover, there is a $\delta \in F I(X) \cap S$. Then there is a $\eta \in A$ with ker $\alpha=$ ker $\eta$ and $i m \eta \# \operatorname{ker} \delta$. Since $d(\alpha)=d(\delta)=\aleph_{0}$, there is a bijection $f: D(\delta) \rightarrow D(\alpha)$. We take the $\gamma_{3} \in \operatorname{Sym}(X)$ defined by $i \gamma_{3}=f(i)$ for $i \in D(\delta)$ and $i \gamma_{3}=i \delta^{-1} \eta^{-1} \alpha$ for $i \in i m \delta$. Then for $i \in X$, $i \eta \delta \gamma_{3}=\left(i \eta \delta \delta^{-1} \eta^{-1}\right) \alpha=i \alpha$ since $i m \eta \# \operatorname{ker} \delta$. This shows $\alpha=\eta \delta \gamma_{3}$ and consequently, $B \subset S$. Altogether, $\operatorname{Inf}(X)=A \cup B \subseteq S$ and thus $S=T(X)$.

Lemma 7. Let $\operatorname{Inj}(X) \subset S \leq T(X)$ with $H \cap S \neq \emptyset$ for $H \in\left\{\operatorname{Sur}(X), C_{p}(X), I F(X)\right\}$. Then

$$
S=T(X)
$$

Proof. We show that then $\operatorname{Sur}(X) \subset S$. If we have $\operatorname{Sur}(X) \subset S$ then from $\operatorname{Inj}(X) \cap S \neq$ $\emptyset$ and $F I(X) \cap S \neq \emptyset$ (because of $\operatorname{Inj}(X) \subset S$ ) it follow $S=T(X)$ by Lemma 6 .

Let $\alpha \in \operatorname{Sur}(X)$. Moreover, there is a $\beta \in C_{p}(X) \cap S$. Then there is a bijection

$$
f: X / \operatorname{ker} \alpha \rightarrow\left\{x \beta^{-1} \mid x \in K(\beta)\right\} .
$$

For each $\bar{x} \in X / \operatorname{ker} \alpha$, there is an injective mapping

$$
f_{\bar{x}}: \bar{x} \rightarrow f(\bar{x}) .
$$

We take the $\gamma \in \operatorname{Inj}(X)$ with $i \gamma=f_{\bar{x}}(i)$ where $i \in \bar{x}$ for $\bar{x} \in X / \operatorname{ker} \alpha$. There are $\delta \in \operatorname{Sur}(X) \cap S$ and $\eta \in I F(X) \cap S$. If $c(\delta)<\aleph_{0}$ then from $c(\delta)>0$, it follows $c\left(\delta^{r}\right)>d(\eta)$ for some $r \in \mathbb{N}$, where $\delta^{r} \in \operatorname{Sur}(X) \cap S$. Hence, we can assume that $c(\delta)>d(\eta)$ and there is a set $A \subseteq X$ with $A \# \operatorname{ker} \delta$ and a bijection

$$
h_{1}: i m \eta \rightarrow A
$$

and an injective mapping

$$
h_{2}: D(\eta) \rightarrow X \backslash A
$$

We take the $\gamma_{1} \in \operatorname{Inj}(X)$ with $i \gamma_{1}=h_{1}(i)$ for $i \in i m \eta$ and $i \gamma_{1}=h_{2}(i)$ for $i \in D(\eta)$. Clearly, $\eta \gamma_{1} \delta \in \operatorname{Sur}(X) \cap S$ with $c\left(\eta \gamma_{1} \delta\right)=\aleph_{0}$. So, we can assume that $c(\delta)=\aleph_{0}$. For $i, j \in X, i \alpha=j \alpha$ if and only if there is an $\bar{x} \in X / \operatorname{ker} \alpha$ with $i, j \in \bar{x}$, i.e. $f_{\bar{x}}(i) \beta=f_{\bar{x}}(j) \beta$. But $f_{\bar{x}}(i) \beta=f_{\bar{x}}(j) \beta$ is equivalent to $i \gamma \beta=j \gamma \beta$, consequently, we have $i \gamma \beta=j \gamma \beta$ if and only if $i \alpha=j \alpha$. Further, let $B \subseteq X$ with $B \# \operatorname{ker} \delta$ and

$$
\varphi: D(\gamma \beta) \rightarrow X \backslash B
$$

be a injective mapping. Then the transformation $\gamma_{2}$ on $X$ with $i \gamma \beta \gamma_{2}$ is the unique element in $i \alpha \delta^{-1} \cap B$ for $i \in X$ and $i \gamma_{2}=\varphi(i)$ for $i \in D(\gamma \beta)$ belongs to $\operatorname{Inj}(X)$. So, we have $i \gamma \beta \gamma_{2} \delta=i \alpha \delta^{-1} \delta=i \alpha$ for $i \in X$. This shows that $\gamma \beta \gamma_{2} \delta=\alpha$, and consequently, $\operatorname{Sur}(X) \subset S$. 
Now we are able to characterize the maximal subsemigroups of $T(X)$ containing $\operatorname{Inj}(X)$ and $\operatorname{Sur}(X)$, respectively.

Theorem 8. Let $\operatorname{Sur}(X) \subset S \leq T(X)$. Then $S$ is maximal iff $S=T(X) \backslash \operatorname{Inj}(X)$ or $S=T(X) \backslash F I(X)$.

Proof. By Proposition 4, both $T(X) \backslash \operatorname{Inj}(X)$ and $T(X) \backslash F I(X)$ are maximal subsemigroups of $T(X)$. Suppose that $S$ is a maximal subsemigroup of $T(X)$. Then $\operatorname{Inj}(X) \cap S=\emptyset$ or $F I(X) \cap S=\emptyset$ by Lemma 6, i.e. $S \subseteq T(X) \backslash \operatorname{Inj}(X)$ or $S \subseteq T(X) \backslash F I(X)$ and thus $S=T(X) \backslash \operatorname{Inj}(X)$ or $S=T(X) \backslash F I(X)$ because of the maximality of $S$.

Theorem 9. Let $\operatorname{Inj}(X) \subset S \leq T(X)$. Then $S$ is maximal iff $S=T(X) \backslash H$ for some $H \in\left\{\operatorname{Sur}(X), C_{p}(X), I F(X)\right\}$.

Proof. By Proposition 4, $T(X) \backslash H\left(H \in\left\{\operatorname{Sur}(X), C_{p}(X), I F(X)\right\}\right)$ are maximal subsemigroups of $T(X)$. If $S$ is a maximal subsemigroup of $T(X)$ then $H \cap S=\emptyset$ for some $H \in\left\{\operatorname{Sur}(X), C_{p}(X), I F(X)\right\}$ by Lemma 7, i.e. $S \subseteq T(X) \backslash H$ for some $H \in\left\{\operatorname{Sur}(X), C_{p}(X), I F(X)\right\}$. The maximality of $S$ provides the assertion.

Finally, we want to determine the maximal subsemigroups of $T(X)$ containing $H$ for $H \in\left\{C_{p}(X), I F(X), F I(X)\right\}$ using Theorem 3. First we state that $F I(X)$ as well as $I F(X)$ are subsemigroups of $T(X)$.

Lemma 10. $F I(X)$ is a subsemigroup of $T(X)$.

Proof. Let $\alpha, \beta \in F I(X)$. Then we have $c(\alpha \beta) \leq c(\alpha)+c(\beta)<\aleph_{0}+\aleph_{0}=\aleph_{0}$ and $\aleph_{0}=d(\beta) \leq d(\alpha \beta)$. This shows that $\alpha \beta \in F I(X)$.

Lemma 11. $I F(X)$ is a subsemigroup of $T(X)$.

Proof. Let $\alpha, \beta \in I F(X)$. Then we have $d(\alpha \beta) \leq d(\alpha)+d(\beta)<\aleph_{0}+\aleph_{0}=\aleph_{0}$ and $\aleph_{0}=c(\beta) \leq c(\alpha \beta)$. This shows that $\alpha \beta \in I F(X)$.

Let us consider the set $C_{p}(X) \cap S u r(X)$. Then we have:

Lemma 12. We have $\langle F I(X), \alpha\rangle=T(X)$ for all $\alpha \in C_{p}(X) \cap S u r(X)$.

Proof. Let $\alpha \in C_{p}(X) \cap \operatorname{Sur}(X), \beta \in \operatorname{Inj}(X)$, and $A \subseteq X$ be a transversal of $\alpha$. We put $\gamma \in T(X)$ setting $x \gamma$ is the unique element in $x \beta \alpha^{-1} \cap A$ for all $x \in X$. It is easy to verify that $i m \gamma \subseteq A$ and $d(\gamma)=|X \backslash i m \gamma| \geq|X \backslash A|=\aleph_{0}$. Let $x, y \in X$ with $x \gamma=y \gamma$. This implies $\left(x \beta \alpha^{-1} \cap A\right) \alpha=\left(y \beta \alpha^{-1} \cap A\right) \alpha, x \beta=y \beta$, and $x=y$ since $\beta \in \operatorname{Inj}(X)$. Thus $\gamma \in \operatorname{Inj}(X)$ and $c(\gamma)=0<\aleph_{0}$. Consequently, $\gamma \in F I(X)$. Because of $x \gamma \alpha=\left(x \beta \alpha^{-1} \cap\right.$ $A) \alpha=x \beta$ for all $x \in X$, we have $\beta=\gamma \alpha \in\langle F I(X), \alpha\rangle$. This shows that $\operatorname{Inj}(X) \subseteq$ $\langle F I(X), \alpha\rangle$. Moreover, $\langle F I(X), \alpha\rangle \cap H \neq \emptyset$ for $H \in\left\{\operatorname{Sur}(X), C_{p}(X), I F(X)\right\}$. By Lemma 7, we have $\langle F I(X), \alpha\rangle=T(X)$.

Lemma 10, Lemma 12 and Theorem 3 imply: 
Proposition 13. Let $S \leq T(X)$ with $F I(X) \subseteq S$. Then the following statements are equivalent:

(i) $S$ is maximal.

(ii) $S=T(X) \backslash H$ for some $H \in \mathcal{H}\left(C_{p}(X) \cap S u r(X), F I(X)\right)$.

Now, we consider the set $F I(X) \cup \operatorname{Inj}(X)$. Here, we get:

Lemma 14. We have $\langle\operatorname{IF}(X), \alpha\rangle=T(X)$ for all $\alpha \in F I(X) \cap \operatorname{Inj}(X)$.

Proof. Let $\alpha \in F I(X) \cap \operatorname{Inj}(X)$ and $\beta \in \operatorname{Inj}(X)$. We put $\gamma \in T(X)$ setting

$$
\begin{gathered}
x \alpha \gamma:=x \beta \text { for } x \in X \\
x \gamma:=f(x) \text { for } x \in D(\alpha)
\end{gathered}
$$

where

$$
f: D(\alpha) \rightarrow D(\beta) \cup x_{0} \alpha
$$

is a surjective transformation such that $|D(\alpha) \backslash \Sigma|=\aleph_{0}$ for some transversal $\Sigma$ of $f$ and any fixed $x_{0} \in X$. Such a mapping exists because of $d(\alpha)=\aleph_{0}$. Since $c(f)=\aleph_{0}$, we have $c(\gamma)=\aleph_{0}$. Moreover, $i m \gamma=\{x \gamma \mid x \in X\}=\{x \gamma \mid x \in i m \alpha\} \cup\{x \gamma \mid x \in$ $X \backslash i m \alpha\}=i m \beta \cup(X \backslash i m \beta) \cup\left\{x_{0} \alpha\right\}=X$. Hence $d(\gamma)=0$. This shows that $\gamma \in \operatorname{IF}(X)$. By definition, we have $\beta=\alpha \gamma \in\langle\operatorname{IF}(X), \alpha\rangle$. This shows that $\operatorname{Inj}(X) \subseteq\langle\operatorname{IF}(X), \alpha\rangle$. Moreover, $\langle\operatorname{IF}(X), \alpha\rangle \cap H \neq \emptyset$ for $H \in\left\{\operatorname{Sur}(X), C_{p}(X), I F(X)\right\}$. By Lemma 7, we have $\langle I F(X), \alpha\rangle=T(X)$.

Lemma 11, Lemma 14 and Theorem 3 imply:

Proposition 15. Let $S \leq T(X)$ with $I F(X) \subseteq S$. Then the following statements are equivalent:

(i) $S$ is maximal.

(ii) $S=T(X) \backslash H$ for some $H \in \mathcal{H}(\operatorname{Inj}(X) \cap F I(X), I F(X))$.

Lemma 16. $\left\langle C_{p}(X)\right\rangle \cap(\operatorname{Inj}(X) \cap F I(X))=\emptyset$.

Proof. Let $\alpha, \beta \in T(X)$ with $c(\alpha)=c(\beta)=\aleph_{0}$. Then $\aleph_{0}=c(\alpha) \leq c(\alpha \beta)$, i.e. $c(\alpha \beta)=$ $\aleph_{0}$. Since $c(\alpha)=\aleph_{0}$ for all $\alpha \in C_{p}(X)$, this shows that $\left\langle C_{p}(X)\right\rangle \cap F I(X)=\emptyset$.

Since we can decompose a countable set into countable many countable sets, it is routine that each transformation $\alpha$ with $\exists \bar{x} \in X / \operatorname{ker} \alpha$ with $|\bar{x}|=\aleph_{0}$ can be written as product $\beta \gamma$ of appropriate transformations $\beta, \gamma \in C_{p}(X)$. Moreover, it is clear that $\left\{\alpha \in T(X) \mid \exists \bar{x} \in X /\right.$ ker $\alpha$ with $\left.|\bar{x}|=\aleph_{0}\right\}$ is subsemigroup of $T(X)$. Hence $\left\langle C_{p}(X)\right\rangle=$ $\left\{\alpha \in T(X) \mid \exists \bar{x} \in X /\right.$ ker $\alpha$ with $\left.|\bar{x}|=\aleph_{0}\right\}$.

Lemma 17. We have $\left\langle C_{p}(X), \alpha\right\rangle=T(X)$ for all $\alpha \in F I(X) \cap \operatorname{Inj}(X)$.

Proof. We show that $\operatorname{Inj}(X) \subset\left\langle C_{p}(X), \alpha\right\rangle$. If we have it then from $\left\langle C_{p}(X), \alpha\right\rangle \cap H \neq \emptyset$ for $H \in\left\{\operatorname{Sur}(X), C_{p}(X), I F(X)\right\}$ it follows $\left\langle C_{p}(X), \alpha\right\rangle=T(X)$ by Lemmal7 For this let $\beta \in \operatorname{Inj}(X)$. Let $\alpha \in S \cap(F I(X) \cap \operatorname{Inj}(X))$. Further let $\left\{I_{k} \mid k \in X\right\}$ be a decomposition of $D(\alpha)$ in infinitely many infinite subsets. Then we take $\gamma \in C_{p}(X) \subset\left\langle C_{p}(X), \alpha\right\rangle$ with $X^{+} / \operatorname{ker} \gamma=\left\{I_{k} \cup\{k \alpha\} \mid k \in X\right\}$ and $i \gamma=k \beta$ for $i \in I_{k} \cup\{k \alpha\}$ and $k \in X$. This provides $k \alpha \gamma=k \beta$ for $k \in X$. This shows $\beta=\alpha \gamma \in S$. Consequently, $\operatorname{Inj}(X) \subset\left\langle C_{p}(X), \alpha\right\rangle$. 
Proposition 18. Let $S \leq T(X)$ with $C_{p}(X) \subseteq S$. Then the following statements are equivalent:

(i) $S$ is maximal.

(ii) $S=T(X) \backslash H$ for some $H \in \mathcal{H}\left(F I(X) \cap \operatorname{Inj}(X),\left\langle C_{p}(X)\right\rangle\right)$.

\section{Acknowledgments}

We gratefully acknowledge the financial support provided for this research by Faculty of Science under grant $\sharp$ RGP 2554-10.

\section{References}

[1] J. East, J. D. Mitchell, Y. Presse, Maximal Subsemigroups of the Semigroup of all Mappings on an Infinite Set, arXiv:1104.2011V2

[2] O. Ganyushkin and V. Mazorchuk, Classical Finite Transformation Semigroups, Springer-Verlag, London, 2009.

[3] L. Heindorf, The Maximal Clones on Countable Sets that include all permutations, Algebra Universalis, 48(2) (2002), 209-222.

[4] P.M. Higgins, J.M. Howie, and N. Ruškuc, On Relative Ranks of Full Transformation Semigroups, Comm. Algebra 26 (1998), 733-748.

[5] J. M. Howie, Fundamentals of Semigroup Theory, Oxford University Press, 1995.

[6] M. Pinsker, Maximal Clones on uncountable sets that include all permutations, Algebra Universalis, 54(2) (2005), 129-148. 